\title{
Modeling metastasis biology and therapy in real time in the mouse lung
}

\author{
Arnulfo Mendoza, ${ }^{1}$ Sung-Hyeok Hong, ${ }^{1}$ Tanasa Osborne, ${ }^{1}$ Mohammed A. Khan, ${ }^{1,2}$ Kirk Campbell, ${ }^{1,2}$ \\ Joseph Briggs, ${ }^{1}$ Ananth Eleswarapu, ${ }^{1,2}$ Lauren Buquo, ${ }^{1}$ Ling Ren, ${ }^{1}$ Stephen M. Hewitt, ${ }^{3}$ \\ EI-H. Dakir, ${ }^{4}$ Susan Garfield, ${ }^{5}$ Renard Walker, ${ }^{6}$ Glenn Merlino, ${ }^{6}$ Jeffrey E. Green, ${ }^{6}$ \\ Kent W. Hunter, ${ }^{6}$ Lalage M. Wakefield, ${ }^{6}$ and Chand Khanna ${ }^{1}$
}

\begin{abstract}
${ }^{1}$ Tumor and Metastasis Biology Section, Pediatric Oncology Branch, Center for Cancer Research, National Cancer Institute, NIH, and ${ }^{2}$ Howard Hughes Medical Institute/NIH Research Scholars Program, Bethesda, Maryland, USA. ${ }^{3}$ Tissue Array Research Program, Laboratory of Pathology, Center for Cancer Research, National Cancer Institute, ${ }^{4}$ Cell and Cancer Biology Branch, Center for Cancer Research, National Cancer Institute, ${ }^{5}$ Center for Cancer Research Confocal Microscopy Core Facility, Laboratory of Experimental Carcinogenesis, National Cancer Institute, and ${ }^{6}$ Laboratory of Cancer Biology and Genetics, Center for Cancer Research, National Cancer Institute, NIH, Bethesda, Maryland, USA.
\end{abstract}

\begin{abstract}
Pulmonary metastasis remains the leading ca use of death for cancer patients. Opportunities to improve treatment outcomes for patients require new methods to study and view the biology of metastatic progression. Here, we describe an ex vivo pulmonary metastasis assay (PuMA) in which the metastatic progression of GFPexpressing cancer cells, from a single cell to the formation of multicellular colonies, in the mouse lung microenvironment was assessed in real time for up to 21 days. The biological validity of this assay was confirmed by its prediction of the in vivo behavior of a variety of high- and low-metastatic human and mouse cancer cell lines and the discrimination of tumor microenvironments in the lung that were most permissive to metastasis. Using this approach, we provide what we believe to be new insights into the importance of tumor cell interactions with the stromal components of the lung microenvironment. Finally, the translational utility of this assay was demonstrated through its use in the evaluation of therapeutics at discrete time points during metastatic progression. We believe that this assay system is uniquely capable of advancing our understanding of both metastasis biology and therapeutic strategies.
\end{abstract}

\section{Introduction}

Pulmonary metastasis remains a leading cause of death for cancer patients $(1,2)$. Opportunities to improve outcomes for these patients require a greater understanding of the biology of metastasis. In addition, there is a need to evaluate novel therapeutics, in a timely manner, that specifically target metastases and metastatic progression. Simple in vitro assay systems are not sufficient to model the complex interaction between cancer cells and the surrounding microenvironment that is necessary for metastasis (3). Accordingly, in vivo models of metastasis, largely in mice, have been necessary. For the most part, these models provide end points of metastatic outcome (i.e., yes or no metastasis) and time to late-stage metastatic events.

A "black box" exists during which metastatic progression from single cells to gross metastatic lesions at a secondary site occurs. Recent attempts to shed light on this process have included imaging strategies that allow some of the steps of metastatic progression to be followed in vivo (4). However, these approaches often involve sophisticated and expensive imaging techniques that are time consuming and do not easily allow serial assessment of early metastatic progression at secondary sites, particularly in the lung and at the single-cell level. Challenges associated with studying metastasis have resulted in limited opportunities to include the assessment of novel treatment agents against metastatic end points (5). Therefore, an unmet need in the field of cancer research is a simple assay in which the process of metastatic progression at a secondary site can be reproduced and studied over time.

Authorship note: Arnulfo Mendoza and Sung-Hyeok Hong contributed equally to this work.

Conflict of interest: The authors have declared that no conflict of interest exists. Citation for this article: J Clin Invest. 2010;120(8):2979-2988. doi:10.1172/JCI40252.
An optimal assay would recapitulate the cellular and microenvironmental complexity of the metastatic site within a native $3 \mathrm{D}$ architecture, while allowing an "open window" for evaluation of metastatic progression. With this goal in mind, we have developed an ex vivo pulmonary metastasis assay (PuMA) in which GFPexpressing cancer cells proliferate and progress in lung tissue. This assay allows real-time assessment of progression from single metastatic cells to multicellular colonies in the lung. This assay faithfully discriminates between high- and low-metastatic phenotypes of human and murine cancer cell lines and between lung (host) microenvironments most permissive to metastasis, demonstrating the relevance and value of the approach. Finally, the assay can be easily scaled to allow for rapid screening of novel therapeutic agents at several dose and schedule combinations. Using this assay, we provide new data that support the importance of tumor cell interaction with stromal elements in the lung microenvironment as a critical determinant of the metastatic phenotype of cancer. The description and validation of this assay immediately provide researchers an opportunity to explore mechanisms for cancer progression at secondary sites and to optimally develop novel treatment approaches specific to cancer metastasis.

\section{Results}

PuMA. We report herein on a metastasis assay that allows real-time assessment of metastatic progression in ex vivo cultures of lung tissue (Figure 1). Using the reported assay conditions, the lung architecture was maintained for over 21 days (Figure 2) and provided a $3 \mathrm{D}$ collagen network with associated lung epithelial cells, inflammatory cells, and other stromal elements upon which fluorescent metastatic cells interacted and then progressed to form metastatic colonies (Figures 3 and 4). Routine histological examination (Figure 2A), 
A

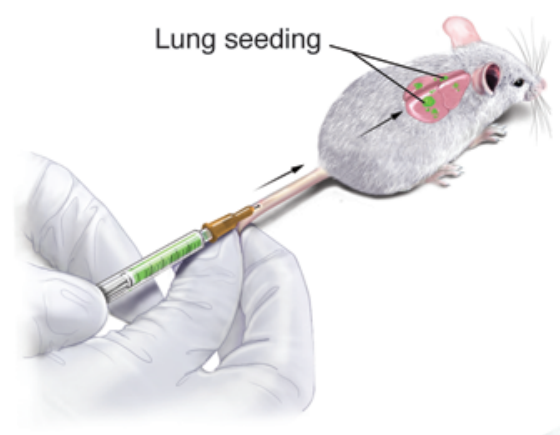

C

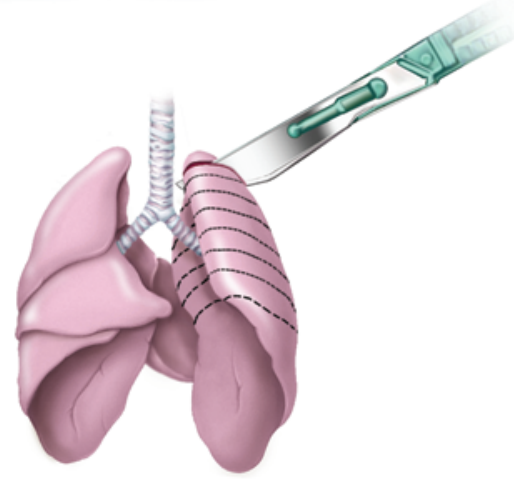

B

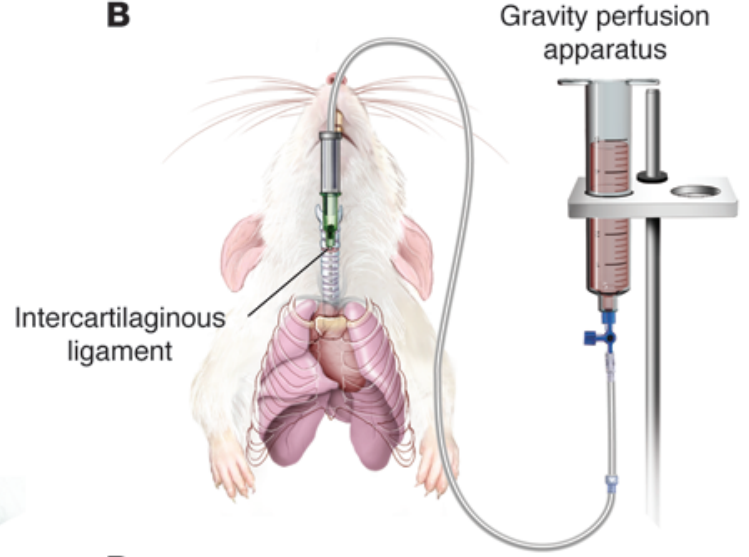

$\mathbf{E}$
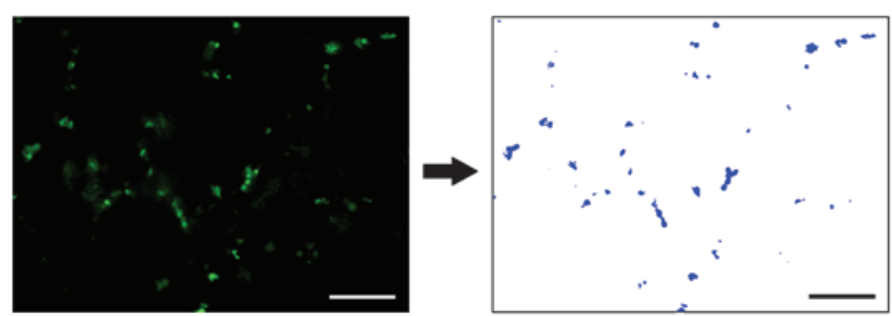

\section{Figure 1}

Schematic diagram summarizing the PuMA experimental approach. (A) GFP-positive tumor cells $\left(2 \times 10^{5}\right)$ were delivered to mice by tail-vein injection. (B) Following humane euthanasia, the trachea was cannulated with 20 -gauge intravenous catheter and attached to a gravity perfusion apparatus. The lungs were infused in the vertical position under a constant $20 \mathrm{~cm} \mathrm{H}_{2} \mathrm{O}$ hydrostatic pressure. (C) The lungs were allowed to cool at $4^{\circ} \mathrm{C}$ for 20 minutes to solidify the agarose medium solution. Complete transverse serial sections (1-2 mm in thickness) were gently sliced from each lobe with a scalpel, yielding 16-20 lung slices per pair of lungs. (D) 4-5 lung sections were placed on the sterile Gelfoam sections bathing in culture media. (E) Images were acquired and the area of GFP-positive cells in each lung was quantified. Scale bars: $200 \mu \mathrm{m}$.

Movat pentachrome histochemical stains for connective tissue components (Figure 2B), and electron microscopy (Figure 2C) confirmed the maintenance of physiologically relevant collagen lung architecture over a 21-day period of ex vivo PuMA (Figure 2). Confocal second harmonic generation (SHG) imaging of lung tissues confirmed the maintenance of a 3D collagen lattice in lung tissues (Supplemental Figure 1; supplemental material available online with this article; doi:10.1172/JCI40252DS1). Cell types present in the PuMA at early time points following lung culture included migratory cells, type I and type II pneumocytes, alveolar macrophages, vascular endothelial cells, red blood cells, airway-associated epithelial cells, and stromal cells. The number and viability of some cellular populations declined through 21 days of culture (Figure 2).

Serial assessment of metastatic progression from single cells in PuMA. Fluorescent metastatic cells were identified microscopically and images were captured to quantify single metastatic cells and metastatic clusters using epifluorescence or confocal microscopy (Figures 1 and 4; Supplemental Figure 1). To validate the utility of the PuMA for the study of metastatic progression, we first compared previously described highly metastatic versus nonmetastatic clonally related human (6) and murine (7) osteosarcoma cell lines. Osteosarcoma is a highly metastatic pediatric cancer with a high proclivity for lung metastasis. These cell lines have similar in vitro growth properties and equally form primary tumors in mice, yet show distinctive in vivo phenotypes in both experimental (i.e., tail-vein injection) and spontaneous (i.e., orthotopic injection) metastasis models in mice. As shown in Figure 4 (and Supplemental Figure 2), the number of metastatic cells that arrive in the lung with the high- and low-metastatic cells is similar at early time points. Indeed, differences in the metastatic phenotype only became evident by day 7 in PuMA, with the highly metastatic cells forming small multicellular colonies (approximately 10-15 
A

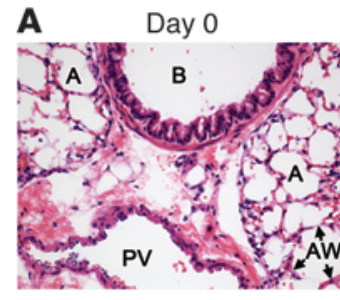

\section{B}
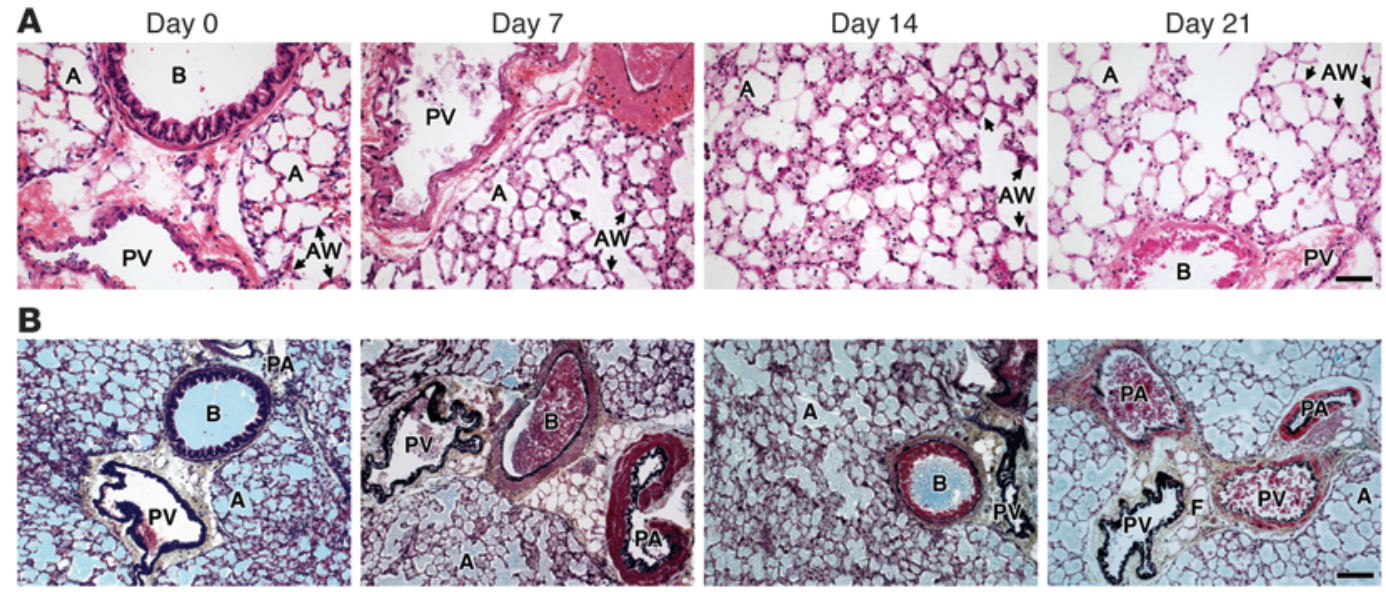

C
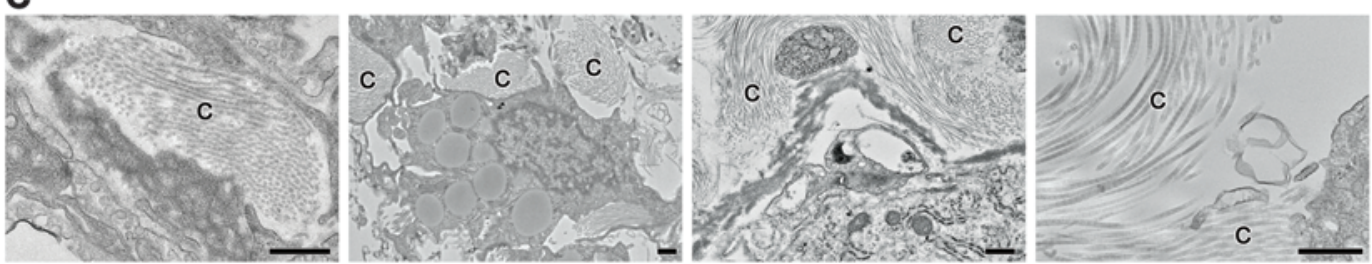

\section{Figure 2}

Demonstration of a viable and structurally intact pulmonary architecture in PuMA. (A) PuMA yields an intact lung microarchitecture. Day 0: bronchioles (B) were lined by epithelia that contact the basement membrane. Alveoli (A) were uniformly expanded throughout the lung, and the alveolar walls (AW, with arrows) were normal in diameter. The alveolar walls contained small numbers of migratory inflammatory cells, pneumocytes (type I and II), and endothelial cells. Blood vessels and alveolar capillaries were expanded by rbcs. Day 7: alveoli remained expanded. There were decreased numbers of migratory cells, pneumocytes, and endothelial cells in the alveolar walls, and many of those that remained contained pyknotic nuclei. Days 14 and 21: alveoli, airways, and large vessels (PA, pulmonary arteries; PV, pulmonary veins) remained expanded. Alveolar capillaries and rbcs were no longer discernible, and the alveolar walls contained fewer migratory cells and pneumocytes (loss of cellularity). Overall, lung microarchitecture was remarkably unchanged. (B) Movat stain was used to examine the connective tissue components of the lung culture. Black elastin fibers were present in large vessels, in the basement membrane supporting the airway epithelia, and within the alveolar interstitium. Black nuclei were scattered throughout the alveolar interstitium. Red muscle surrounded arteries and larger airways (B) and yellow collagen fibers were in the surrounding vascular submucosa and alveolar interstitium. Each of these components was identified at each time point. (C) TEM. Stromal elements composed of collagen microfibers (C) were evident from day 0 through day 21 . Scale bars: $100 \mu \mathrm{m}$ (A and B); $1 \mu \mathrm{m}$ (C).

cells), whereas the clonally related, nonmetastatic cells formed few multicellular clusters and then showed progressive decline in the number of single metastatic cells. The localization of single tumor cells was visualized by merging fluorescent images of metastatic cells with confocal SHG reconstruction of the collagen matrix of the lung (Supplemental Figure 1). By day 14, large metastatic colonies were evident in the lung tissue containing highly metastatic osteosarcoma cell lines, whereas very few cells could be identified in lung tissue containing low-metastatic cells (Figure 4, A-D). Interestingly, similar to our observations from in vivo studies of metastasis in mice, most metastatic colonies were observed at the periphery of the lung sections.

In order to demonstrate that the metastatic tumor cells were actively proliferating in the lung cultures, Ki-67 immunohistochemical staining was conducted during the culture period (Figure 3). Indeed, positive Ki-67 staining was observed in metastatic tumor cells in the PuMA. This included single metastatic cells early after their arrival in the lung and in larger metastatic colonies at later time points. In addition, Ki-67 immunoreactivity was observed in the alveolar interstitium, demonstrating the viability of alveolar interstitial cells in this assay. As further validation that metastatic tumor cell numbers increased over time in PuMA, quantitative real-time PCR for the eGFP transgene was performed on genomic DNA isolated from lung slices containing metastatic tumor cells at multiple time points. As expected, the eGFP DNA copy number increased over time, indicative of increased tumor cell number and consistent with fluorescent evaluation showing metastatic progression (Supplemental Figure 3).

To further validate the utility of the assay in the study of metastasis biology, we examined a larger series of clonally related cancer cell lines with known differences in metastatic phenotype (Supplemental Figure 4, with summary in Table 1) $(6,8-13)$. The kinetics of metastatic progression in the PuMA were distinct for each cancer cell line assessed (Figure 4 and Supplemental Figure 4). Nonetheless, in each case, the clonally related variants with greater propensity for metastasis in vivo were also associated with greater metastatic phenotype in the PuMA. It is important to note that metastatic phenotypes of these cell line pairs are indistinguishable in conventional 2D culture conditions (Table 1).

A direct comparison of the PuMA with in vivo experimental metastasis using identical cell lines and assessment points suggested that the kinetics of metastatic progression in the PuMA and in vivo are similar (Figure 5). The primary difference between PuMA and in vivo experimental metastasis was the 
$\underline{A}$ Control

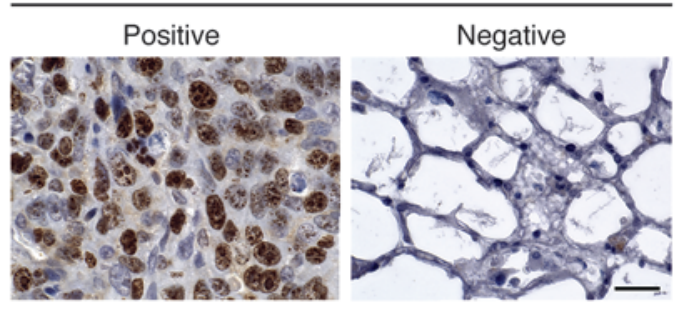

B

Day 0
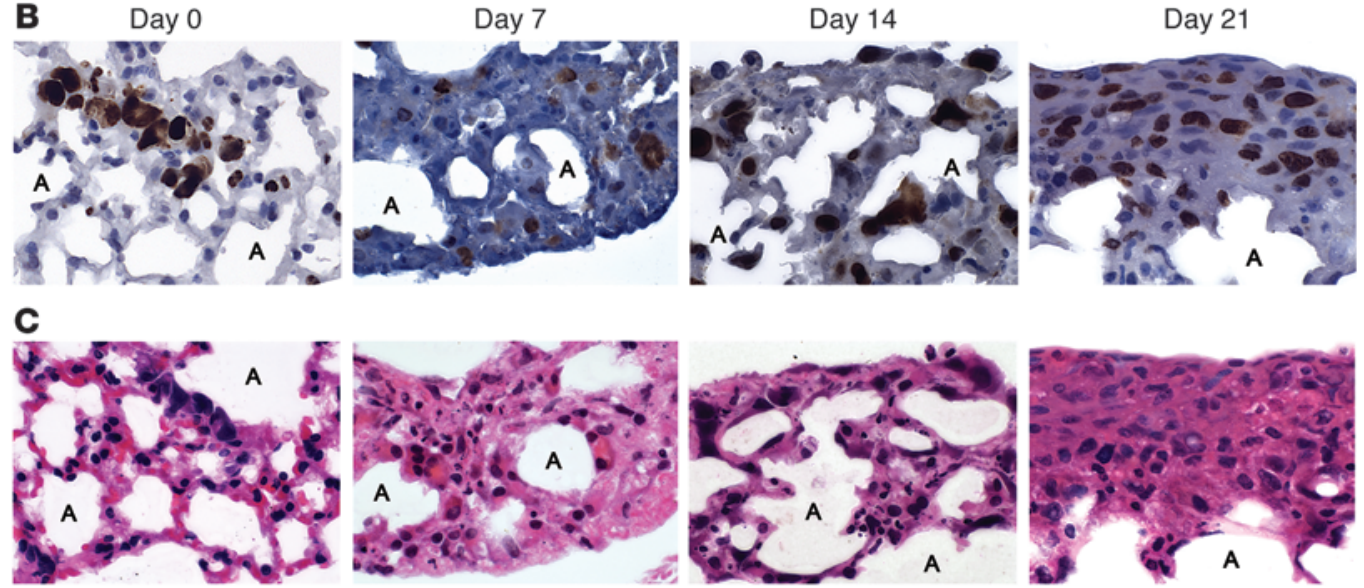

\section{Figure 3}

Immunohistochemical staining of highly metastatic osteosarcoma cells for Ki-67 in PuMA. (A) Positive and negative control, (B) Ki-67 immunohistochemical staining, and (C) H\&E staining of PuMA sections confirmed proliferative competency of metastatic osteosarcoma cells, most notably at days 14 and 21. Tissue from a primary osteosarcoma lesion was used as a positive control for Ki-67. Scale bar: $100 \mu \mathrm{m}$.

duration of the initial period of metastatic inefficiency, during which the number of single metastatic cells detected in the lung was reduced. In the in vivo experimental metastasis setting, this period of inefficiency for osteosarcoma cells was most notable at day 7. In the PuMA, the metastatic inefficiency, for these osteosarcoma cells, was most notable at day 4 (Supplemental Figure 5) and had begun to recover at day 7. Similar and more extreme patterns of metastatic inefficiency were seen in both PuMA and the in vivo setting using nonmetastatic cells (data not shown). Beyond the calibration between PuMA and the in vivo settings, these data suggest that a primary determinant of early metastatic inefficiency is survival of metastatic cells in the lung and is not merely a function of blood flow "washing away" metastatic cells from the lung.

Since the PuMA includes cellular and stromal elements of the host, we next asked whether this system might also allow microenvironmental (i.e., host) influences on metastatic progression to be assessed. AKR/J and DBA/2J represent murine genetic backgrounds in which primary tumor initiation and growth kinetics have been shown to be identical, but where there is a 20 -fold difference in metastatic propensity (13). As predicted by in vivo studies, a more aggressive pattern of metastatic progression was seen for mammary cancer cells in the AKR/J as compared with DBA/2J mouse lung sections (Figure 6 and Table 1). These results support the hypothesis that metastasis-associated differences in genetic background that have been previously reported are indeed the result of differences in the local tumor microenvironment (i.e., lung microenvironment). Furthermore, given the absence of a systemic immune component in the PuMA, these data suggest the importance of stromal elements of the extracellular matrix in the lung as primary determinants of the role of the tumor microenvironment on metastatic progression. These data further underscore the opportunity to evaluate both tumor and microenvironmental influences linked to metastatic progression within the PuMA assay.

Screening of therapeutic agents with potential activity against metastatic progression and metastases. Finally, we sought to determine whether the PuMA assay could be used to assess agents with potential activity against metastasis. The ability of therapeutic agents to diffuse through the culture media into the Gelfoam material and into the lung section tissues was tested within the PuMA using tumor cells that expressed GFP under the control of a doxycycline-sensitive promoter. Within 24 hours of addition of doxycycline to the culture media of the PuMA, we observed expression of GFP in tumor cells (data not shown).

We then compared the activity of therapeutic agents in PuMA in relation to their activity in conventional transplantable murine models (summarized in Table 2 and Supplemental Figure 6). Evaluated therapeutic agents included conventional cytotoxic chemotherapy (treosulfan) and the prototypic mTOR pathway inhibitor (rapamycin). Consistent with in vivo results (14), rapamycin exposures to the PuMA significantly inhibited metastatic progression at $1 \mu \mathrm{M}$. The anticancer activity of treosulfan, a nontargeted cytotoxic chemotherapy, has been shown in vitro and in vivo against a variety of pediatric sarcoma cell lines (15). Significant activity of these agents was confirmed in the PuMA following its addition early or late during the course of metastatic progression. The throughput and flexibility of the 
A
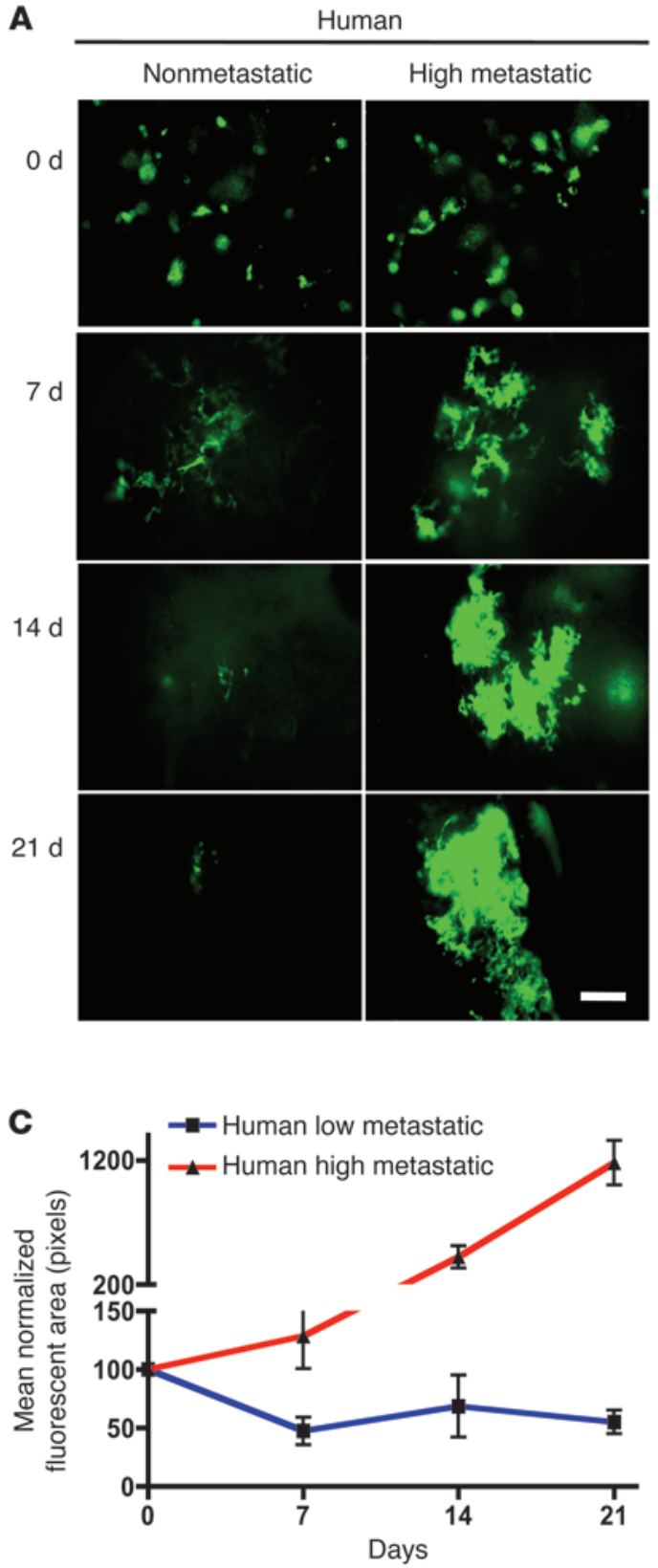

B
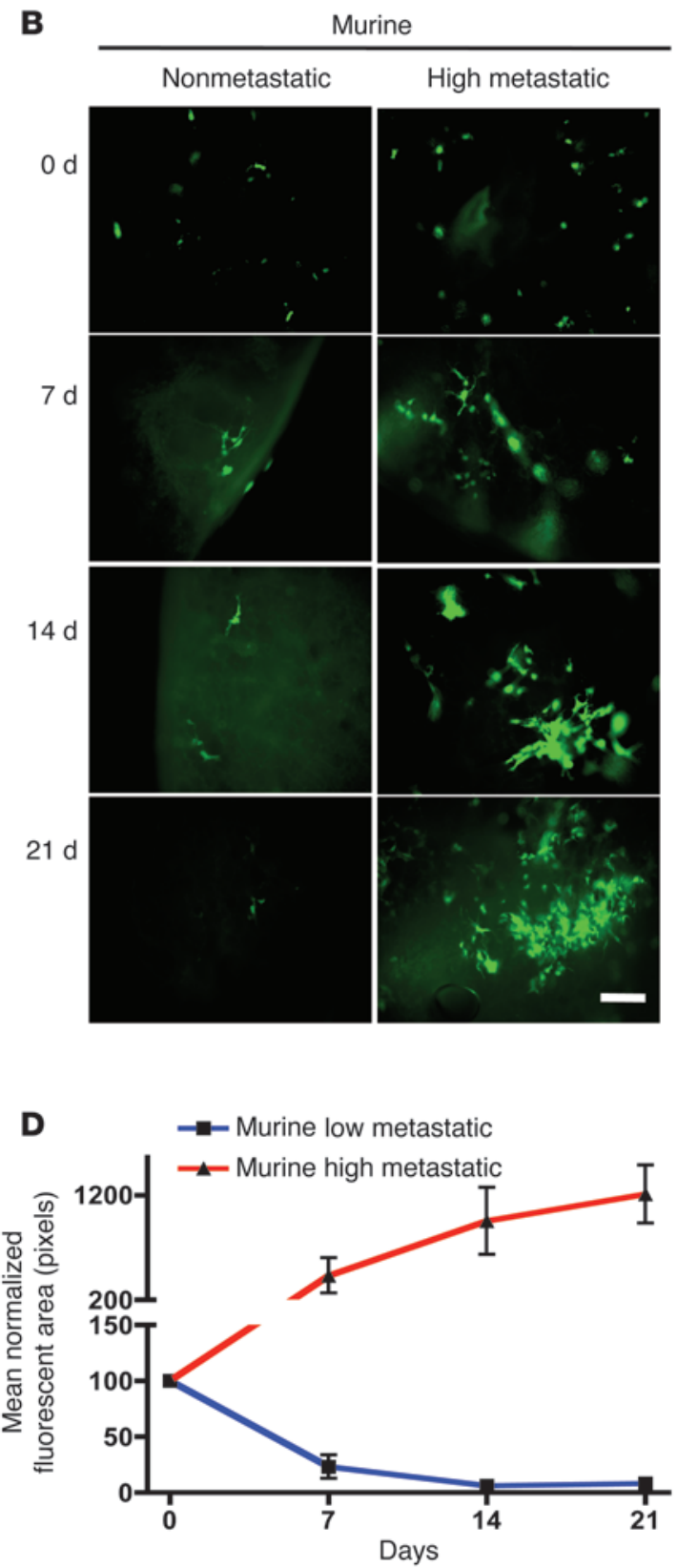

\section{Figure 4}

The PuMA distinguishes the phenotype of metastatic from nonmetastatic cell lines. (A and B) Serial imaging of fluorescently labeled high- and low-metastatic human and murine osteosarcoma cells. Similar numbers of cells were seen at day 0; however, a difference between high- and low-metastatic cells was seen at day 7. Representative fields from lung are shown. Scale bar: $200 \mu \mathrm{m}$. (C and D) Quantification of metastatic burden (mean normalized fluorescent area) from $\mathbf{A}$ and $\mathbf{B}$ reveals a significantly lower sum of GFP-positive cell area in the lung section following injection of low-metastatic human and murine osteosarcoma cells. Similar results were generated using high- and low-metastatic pairs of human and murine breast cancer cells and murine melanoma cells (see Table 1 and Supplemental Figure 4). Quantification of metastatic progression in PuMA was validated using both mean fluorescent area and enumeration of surface metastatic colony count (Supplemental Figure 2). Plotted data represent the mean \pm SD.

PuMA allowed several doses of each agent to be assessed against metastatic progression in a short period of time. The real-time observation provided by the assay allowed the assessment of each agent against either the entire metastatic progression from single metastatic cells to metastatic clusters or at discrete points in the course of metastatic progression in the lung.

\section{Discussion}

The PuMA is an ex vivo closed system and sterile assay that allows assessment of metastatic progression from single cells to metastatic clusters up to and beyond a 21-day observation period. During this time, metastatic tumor cells interact with the 3D collagen network within the lung (the primary constituent of the lung architecture) 


\section{Table 1}

Descriptive summary of concordance between PuMA and in vivo studies of metastasis biology

$\begin{array}{lc}\text { Metastasis biology } & \begin{array}{c}\text { PuMA metastatic phenotype } \\ \text { is discernible }\end{array} \\ \text { Human osteosarcoma (6) } & \text { Yes } \\ \text { Murine osteosarcoma (8) } & \text { Yes } \\ \text { Human breast cancer (9) } & \text { Yes } \\ \text { Murine breast cancer }(10,11) & \text { Yes } \\ \text { Murine melanoma (12) } & \text { Yes } \\ \text { Murine host microenvironments (13) } & \text { Yes }\end{array}$

In vivo metastatic phenotype
is discernible
Yes
Yes
Yes
Yes
Yes
Yes
In vitro metastatic phenotype is discernible ${ }^{c}$
No
No
No
No
No
No

${ }^{A}$ Discernible: can a blinded investigator distinguish metastatic and nonmetastatic cells in the PuMA assay? Refer to Figure 4 and Supplemental Figure 4 for actual experimental data. ${ }^{B}$ Discernible: can a blinded investigator distinguish metastatic and nonmetastatic cells in the in vivo studies? ${ }^{\mathrm{C}} \mathrm{Discernible:} \mathrm{can} \mathrm{a}$ blinded investigator distinguish metastatic and nonmetastatic cells in conventional in vitro tissue culture conditions?

and associated lung epithelial cells, inflammatory cells, and other stromal elements. Fluorescent metastatic cells and metastatic lesions were quantified for number, size, and localization during this period. The relevance of the PuMA assay was confirmed by its ability to accurately define the metastatic phenotype of tumor cell lines with previously established high- versus low-metastatic behaviors in vivo. These predictions are noteworthy since the metastatic phenotype of these cells is not distinguished by growth in conventional in vitro culture conditions. Given the recognized importance of the tumor-microenvironment interactions for metastatic progression, it was particularly revealing to see that the PuMA was used effectively to compare the influences of distinct host microenvironments on the progression of identical tumor cells. These new data suggest the importance of the local microenvironment rather than systemic response of the host on the metastatic phenotype. Finally, the opportunity to use this PuMA approach as a translational drug development tool was then supported by the evaluation of both cytotoxic and molecularly targeted therapeutics against metastatic progression. Importantly, the flexibility of the assay allowed rapid assessment of multiple drug doses, exposure times, and schedules. The PuMA fills an unmet need in the study of metastasis biology and therapy by allowing the real-time assessment of metastatic progression in a relevant tumor microenvironment.

A critical component of the assay conditions reported herein was the insufflation of $0.6 \%$ agarose to the lung (16). Without agarose insufflation, the lung structure was completely lost within 24 hours (data not shown). The influence of agarose density on metastatic tumor cell growth has been previously assessed (16). Conventional microscopy, immunohistochemistry, electron microscopy, and SHG microscopy demonstrated the collagenous nature of the lung architecture. Based on confocal fluorescent microscopy and SHG imaging (which images the collagen matrix of the lung), we hypothesize that the $3 \mathrm{D}$ collagen lattice that is retained in the PuMA is a critical scaffold on which tumor cells interact with other cells of the lung microenvironment. We and others have hypothesized that the survival of tumor cells at distant secondary sites is stressful and that this stress is a primary contribution to the inefficiency of the metastatic process $(17,18)$. Based on the results of the PuMA assay, it is reasonable to argue that metastatic cells have an enhanced capability to engage the local lung microenvironment and manage growth on the scaffolding of the lung collagen architecture than nonmetastatic cells. Indeed, survival in this $3 \mathrm{D}$ context may be the measurement of metastatic competency assessed in this assay. Our studies of differences in metastatic progression in the PuMA that result only from changes in the strain of mice now provide additional information on the importance of the interaction between tumor and tumormicroenvironment as a critical determinant of (strain/host dependent) metastatic success. These data in the PuMA provide a unique perspective on the importance of the noncellular stromal elements of the lung microenvironment in the progression of lung metastasis. It is recognized that different mouse strains express different ECM components. These include those stromal elements observed within the microarray prognosis signatures for metastatic cancers. Based on the PuMA data, we suggest that tumor cells are responding differently to the subtle variations of ECM composition that either induce or suppress growth in the secondary site. These data are concordant with recent reports on the role of lung collagen in regulating the dormancy phenotype of some metastatic cancers (9).

The PuMA provides an opportunity to visualize and monitor the progression of metastasis in the lungs in ways not previously possible. For example, using existing in vivo mouse models, we are unable to easily assess the full range of steps and cellular processes involved in metastatic progression in the lung. This is despite the application of sophisticated imaging approaches to this area of need (19). Furthermore, the PuMA complements recent assay approaches used to monitor metastatic progression, such as of intravital video microscopy (IVVM) (20, 21). Unlike PuMA, IVVM allows the study of metastasis in the context of living tissue with blood flow. IVVM is constrained by a relatively short window of observation, most commonly restricted to hours after cellular arrival at secondary sites. Other effective approaches to image metastatic progression in the lung can be confounded by the impact of surgery needed to expose the lung and the influences of respiring or ventilated lung on image quality. In addition, several simple 3D in vitro culture systems have been used to study the metastatic phenotype of cells. However, these approaches fail to model the complexity of tumor cell/tumor microenvironment interactions necessary for metastasis (22). Our current description of the PuMA addresses many of the shortcomings of currently available methods to study metastasis biology. Indeed, a direct comparison of PuMA with in vivo experimental metastasis demonstrated the similarity in metastatic progression seen in both ex vivo and in vivo conditions. It is reasonable and our expectation that the PuMA will provide opportunities for several future investigations of metastasis biology, including determinants of the metastatic tumor cell, the tumor microenvironment, and their interaction.

Finally, the PuMA assay provides what we believe is a novel approach to specifically evaluate the efficacy of anti-cancer agents in the context of metastatic progression at a secondary 
A

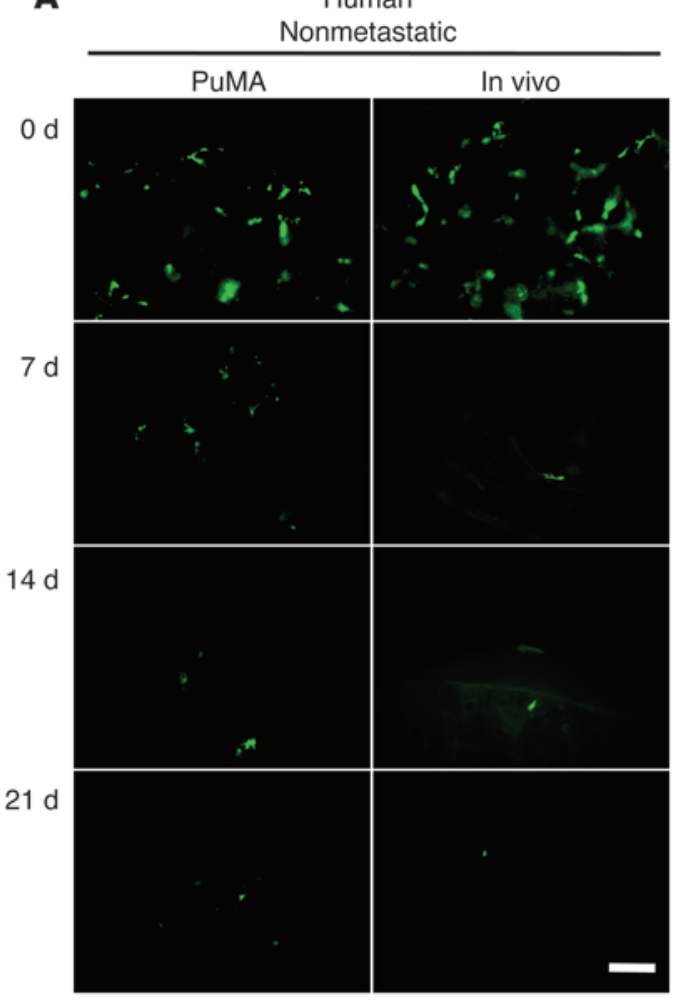

C

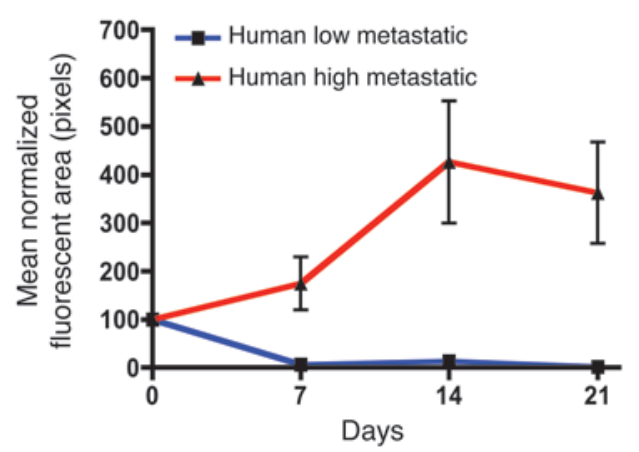

B

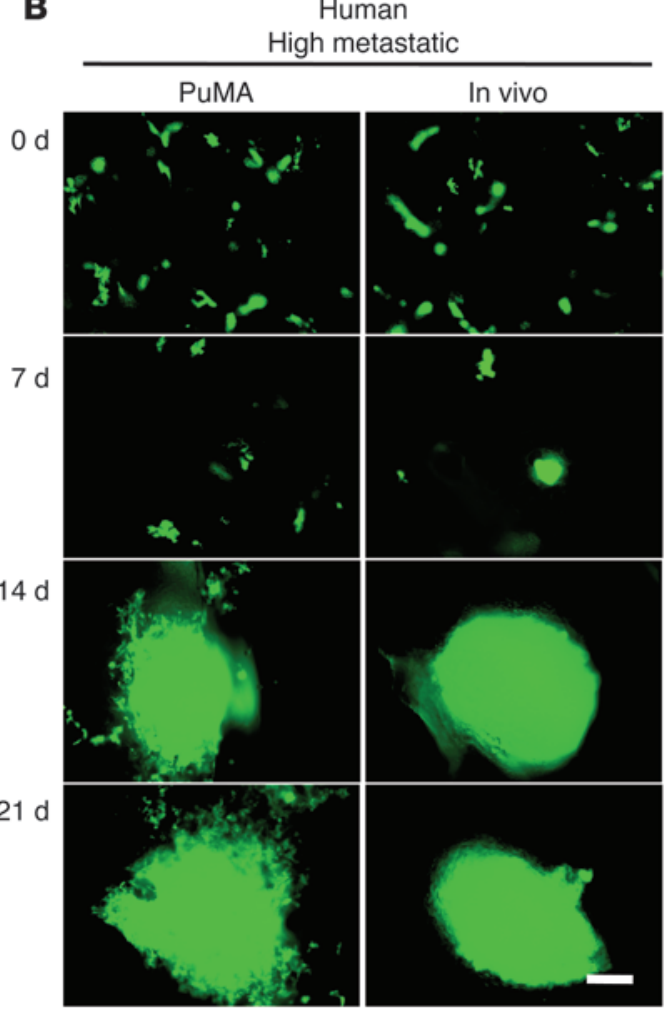

D

In vivo

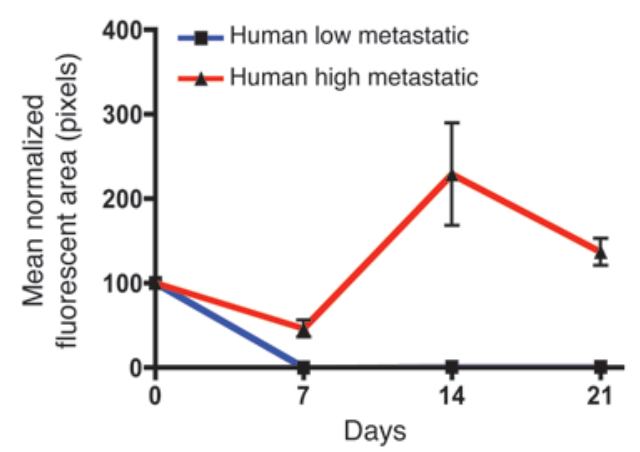

Figure 5

Similarities in metastatic progression in vivo compared with PuMA. A direct comparison of the metastatic phenotype of human osteosarcoma cell lines in vivo (experimental metastasis) and in the PuMA was conducted. (A and B) Serial imaging of fluorescently labeled high- and lowmetastatic human osteosarcoma cells was conducted in the PuMA. At the identical time points, lungs from mice that had received tail-vein injection of tumor cells were collected and imaged as in the PuMA. Patterns of pulmonary metastatic progression were similar in both in vivo and PuMA. Representative fields from lung are shown. Scale bars: $200 \mu \mathrm{m}$. (C and D) Quantification of metastatic burden (mean normalized fluorescent area) from $\mathbf{A}$ and $\mathbf{B}$. Identical results demonstrating the similarities in pulmonary metastatic progression for murine osteosarcoma cells was seen in vivo and in the PuMA. Plotted data represent the mean \pm SD.

site. Despite the fact that the most common cause of death in cancer patients is metastasis, current preclinical drug development models do not prioritize metastasis end points in their evaluation of new drug candidates. This is due in part to the inability to model metastatic progression or metastatic lesions outside the mouse. It is also due to the fact that in vitro assays of specific metastatic processes (i.e., motility or invasion) are, alone, not always informative. Although not a high-throughput option, the PuMA provides an efficient assessment of several drug-dose-schedule combinations over time. Furthermore, the PuMA allows the evaluation of a new drug candidate against either single metastatic cells or advanced metastatic lesions. In this way, the PuMA may be useful in identifying or prioritizing novel cancer therapy agents for use against metastatic progression or metastatic lesions and should therefore accelerate the development of new treatments for patients with metastasis. 
A

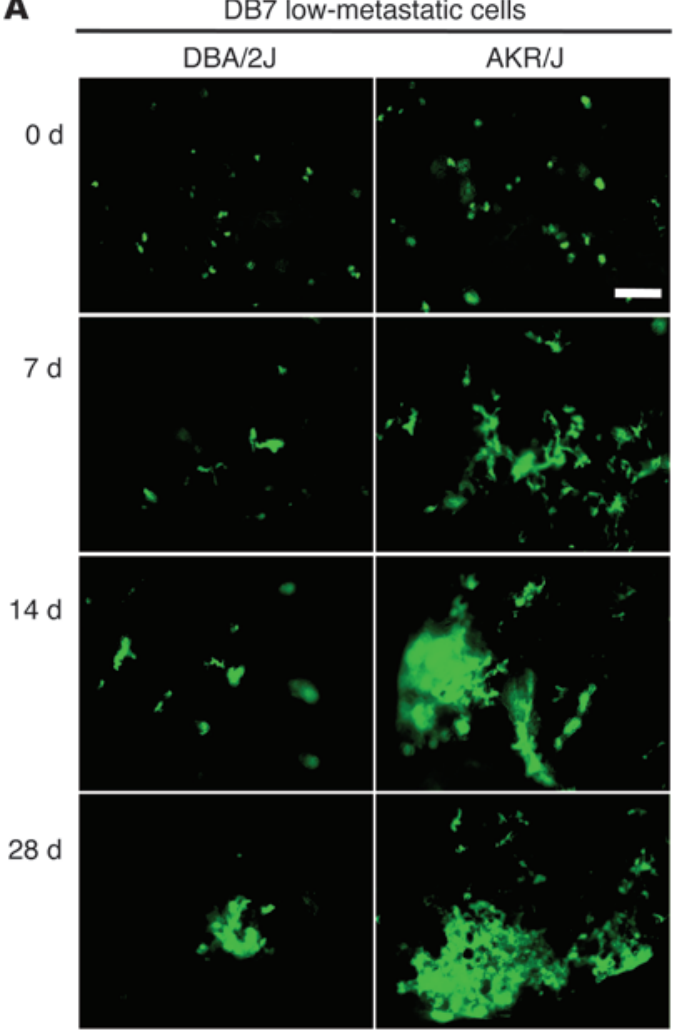

B

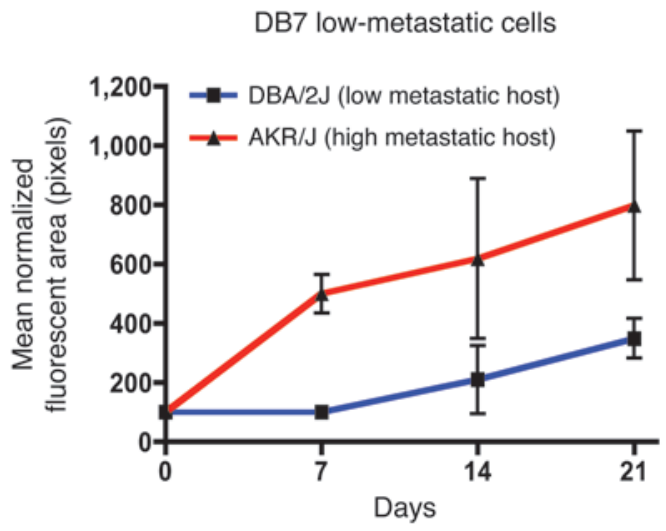

Figure 6

The PuMA can distinguish host microenvironments that are permissive. (A) Serial imaging of fluorescently labeled low-metastatic murine breast cells (DB7) in high- (AKR/J) and low-metastatic (DBA/2J) murine host microenvironments. Similar numbers of metastatic cells are seen at day 0 in both lung environments; however, a difference in metastatic progression between high- and low-metastatic microenvironments can be seen at day 7 . Representative fields from lung are shown. Scale bar: $200 \mu \mathrm{m}$. (B) Reduced metastatic burden (quantified by total normalized fluorescent lung area) was seen in the low-metastatic phenotype of $D A B / 2 J$ lung sections. Total metastatic burden in each lung section was normalized to day 0 and followed over time. Plotted data represent the mean $\pm \mathrm{SD}$.
Although not investigated herein, the PuMA will provide an opportunity to study later points in metastatic progression, i.e., from metastatic colony formation to development of gross metastatic lesions. This may be accomplished by delaying the initiation of the PuMA for days or weeks after the injection of tumor cells to mice. By delaying the initiation of PuMA, the same experimental methodology reported here may also be used following spontaneous metastasis of tumor cells from either orthotopic or heterotopic sites.

The PuMA allows real-time assessment of metastatic progression from single cells in the lung to multicellular colonies. This experimental approach fills an important gap in the field of metastasis research by allowing real-time assessment of metastatic progression from single cells in the lung to multicellular colonies and beyond. The PuMA also provides an informative unique opportunity to study the local host (i.e., stromal) determinants of metastatic progression in cancer. The description and validation of this assay provides investigators with an immediate opportunity to explore mechanisms for cancer progression at secondary sites and to optimally develop novel treatment approaches for cancer metastasis. complete DMEM media (Invitrogen). GFP was expressed in all cells using pSICO-eGFP or p960-X1-685-eGFP lentiviral infection.

Lung organ culture medium. Serum-free conditions used for lung culture were first described by Siminski in 1992 (23) and were modified as follows. Culture medium 1, 2× solution of M-199 (Invitrogen), was supplemented with $2.0 \mu \mathrm{g} / \mathrm{ml}$ crystalline bovine insulin, $0.2 \mu \mathrm{g} / \mathrm{ml}$ hydrocortisone (Sigma-Aldrich), $0.2 \mu \mathrm{g} / \mathrm{ml}$ retinyl acetate (Sigma-Aldrich), $200 \mathrm{U} / \mathrm{ml}$ penicillin and $200 \mu \mathrm{g} / \mathrm{ml}$ streptomycin (Invitrogen), and 7.5\% sodium bicarbonate (Invitrogen). Culture medium 2 was used for media changes during the lung organ culture period; $1 \times$ solution of M-199 was supplemented with $1.0 \mu \mathrm{g} / \mathrm{ml}$ crystalline bovine insulin, $0.1 \mu \mathrm{g} / \mathrm{ml}$ hydrocortisone, $0.1 \mu \mathrm{g} / \mathrm{ml}$ retinyl acetate, $100 \mathrm{U} / \mathrm{ml}$ penicillin and $100 \mu \mathrm{g} / \mathrm{ml}$ streptomycin, and $7.5 \%$ sodium bicarbonate. Then we prepared 1.2\% low-melting agarose solution (FMC BioProducts) with sterile distilled water. The agarose solution was melted by microwave and kept at $40^{\circ} \mathrm{C}$ before use in lung culture. The agarose solution was mixed with culture medium $1\left(37^{\circ} \mathrm{C}\right)$ at a $1: 1$ concentration.

In vivo and ex vivo lung organ culture studies. The overall technique of isolated lung organ culture is schematically shown in Figure 1. Healthy

\section{Methods}

Cell lines. Murine osteosarcoma K7M2 and K7M2/Ezrin antisense 1.46 (8), murine breast cancer 4T07 and 67NR (10), Met1, and DB7 (11), human osteosarcoma HOS and HOSMNNG (6), human breast cancer MCF7/GFP and MDA231/GFP (9), and murine Ink4a/Arf (N-Ras transformed Ink4a/Arf-null, p53knockdown) and N-Ras transformed Ink4anull melanoma cells (12) were cultured in
Table 2

Descriptive summary of the use of PuMA in translational evaluation of anticancer agents with activity against metastasis

$\begin{array}{lccc}\text { Metastasis therapy } & \begin{array}{c}\text { Anticancer activity } \\ \text { in PuMA }\end{array} & \begin{array}{c}\text { Antimetastatic activity } \\ \text { in vivo }\end{array} & \begin{array}{c}\text { Anticancer activity } \\ \text { in vitro }\end{array} \\ \text { Rapamycin (14) } & \text { Yes } & \text { Yes } & \text { Yes } \\ \text { Treosulfan (15) } & \text { Yes } & \text { Yes } & \text { Yes }\end{array}$

Refer to Supplemental Figure 6 for actual experimental data. 
GFP-positive tumor cells $\left(2 \times 10^{5}\right)$ were delivered by tail-vein injection to either female BALB/c (Taconic), SCID/beige (Charles Rivers), FVB/N (Jackson Laboratory), AKR/J- FVB/NJ F1, or DBA/2J - FVB/NJ F1 mice. Within 15 minutes of tumor injection, the mice were euthanized by $\mathrm{CO}_{2}$ inhalation. Using sterile surgical conditions in a laminar flow hood, the mice were placed in dorsal recumbency. The sternum was removed to expose the lung. The trachea was then cannulated with a 20-gauge intravenous catheter (Terumo) and attached to a gravity perfusion apparatus under constant $20 \mathrm{~cm} \mathrm{H}_{2} \mathrm{O}$ hydrostatic pressure or by syringe infusion of $1.2 \mathrm{ml}$ of well-mixed culture medium 1 /agarose solution $\left(40^{\circ} \mathrm{C}\right)$. The trachea, lungs, and heart were then carefully removed and immediately placed in a cold solution of PBS containing $100 \mathrm{U} / \mathrm{ml}$ penicillin and $100 \mu \mathrm{g} / \mathrm{ml}$ streptomycin at $4{ }^{\circ} \mathrm{C}$ for 20 minutes to solidify the agarose/ medium solution. Complete transverse sections (1-2 mm in thickness) were made from each lobe using a \#21 scalpel blade, yielding 16-20 lung sections. Then $4-5$ lung sections were placed on a single $1.5 \times 0.7-\mathrm{cm}$ sterile Gelfoam (Pfizer-Pharmacia \& Upjohn Co.) section that had been preincubated for 2 hours in a 6 -cm tissue culture dish with culture medium 2 . Lung sections were incubated at $37^{\circ} \mathrm{C}$ in humidified conditions of $5 \%$ $\mathrm{CO}_{2}$. Fresh culture medium 2 was replaced and lung tissue sections were turned over with a sterile iris thumb forceps every other day. Drug treatments were added to the culture media at either day 0 (early treatment) or day 21 (late treatment) of the culture period. Animal care and use was in accordance with the guidelines of the NIH Animal Care and Use Committee. All animal studies were approved by the Animal Care and Use Committee of the National Cancer Institute.

Image analysis and quantification. A LEICA-DM IRB fluorescent inverted microscope (Leica) and Retiga-EXi Fast 1394 Mono Cooled CCD camera (QImaging) were used to capture images of GFP-positive tumor cells within the PuMA at $\times 100$ magnification. Fluorescent events within the PuMA were acquired using OpenLab software (Improvision). Metastatic burden was quantified by measuring the fluorescent area of metastatic cells in each lung section at each time point and was expressed as either mean fluorescent area (mean fluorescent area of each lung section over 4 lung sections) or total fluorescent area (sum of fluorescent area in 4 lung sections). Fluorescent area (mean or total) was normalized to 100 pixels for day 0 to allow quantitative evaluation of metastatic progression over time.

Histology/immunohistochemistry. Formalin-fixed, paraffin-embedded mouse lung tissue sections were prepared at $5 \mu \mathrm{m}$ on a transverse plain. Sections were examined using H\&E and special filament stains (cytokeratin and vimentin; data not shown). Movat's pentachrome histochemical staining technique was used to determine the presence of specific connective tissue, muscular, and cellular components. Expected Movat staining results were as follows: nuclei (dark purple to black), elastic fibers (black), muscle and red blood cells (red), collagen and reticular fiber (yellow), mucosubstance (blue to blue-green), and cytoplasm (pink to brownishred) (24). Ki-67 staining was performed using the LSAB + System-HRP kit (Dako North America Inc.) as previously described (25). Purified mouse monoclonal antibody for Ki-67 (1:25; BD Biosciences) was diluted in blocking buffer and incubated overnight at $4^{\circ} \mathrm{C}$. Negative control incubation was performed by substituting nonimmune serum for the primary antibody. Following primary antibody incubation, slides were washed with PBS 3 times and incubated in biotinylated goat anti-mouse IgG secondary antibody (Dako North America, Inc) in 1:500 Dako antibody diluent for 1 hour at room temperature. Streptavidin peroxidase was applied to slides for 30 minutes at room temperature. Color development was achieved by applying a 3-3'-diaminobenzidine chromagen solution. Tissues were counterstained with hematoxylin (Sigma-Aldrich), dehydrated in a series of graded alcohols ending in xylene, mounted with coverslips, and examined by light microscope. Tissues from murine pulmonary metastatic osteosarcoma lesions were used as positive controls for Ki-67.

Transmitted electron microscopy. Mouse lung tissues were fixed in $4 \%$ formaldehyde and $2 \%$ glutaraldehyde (Tousimis) in $0.1 \mathrm{M}$ cacodylate buffer (Electron Microscope Science) and processed using transmitted electron microscopy (TEM) procedures. Briefly, the tissues were washed in cacodylate buffer, post-fixed in osmium tetroxide (1\% in same buffer) (Electron Microscope Science), and en bloc-stained in uranyl acetate $(0.5 \%$ in $0.1 \mathrm{M}$ acetate buffer, $\mathrm{pH}$ 4.2) (Electron Microscope Science). The tissues were dehydrated in a series of ethanol dilutions (e.g., 35\%, 50\%, 75\%, 95\%, and 100\%) followed by propylene oxide and incubated in an equal volume of propylene oxide and epoxy resin overnight. The tissues were embedded in a resin and cured in a $55^{\circ} \mathrm{C}$ oven for 48 hours. The thin-sectioned $(75 \mathrm{~nm})$ samples were mounted on a copper-meshed grid and stained in uranyl acetate (Electron Microscope Science) and lead citrate (Leica) prior to the EM examination. The digital images were taken using a $\mathrm{H} 7600$ microscope (Hitachi) equipped with an AMT camera (Advanced Microscopy Techniques Co.).

Comparison of metastatic progression in PuMA versus in vivo experimental metastasis. Twelve SCID mice received tail-vein injection of the metastatic HOSMNNG human osteosarcoma cell lines on day 0 of the experiment. Lungs from 3 of the mice were processed within the PuMA with image capture on days $0,7,14$, and 21 . Nine mice were followed in vivo. Three mice from this in vivo group were sacrificed and imaged on days 7, 14, and 21 in an identical manner to that used with PuMA. Image analysis and quantification of metastatic progression was identical for both groups.

Additional methods. Descriptions of Confocal SHG microscopy and DNA extraction and quantitative real-time PCR analysis are available in Supplemental Methods.

Statistics. The statistical significance between high-metastatic and lowmetastatic tumor burden was determined by unpaired $t$ test with Welch's correction using Prism (version 4; GraphPad software). $P<0.05$ was considered statistically significant.

\section{Acknowledgments}

We thank K. Nagashima for assistance with EM imaging; Lydia Kibiuk and Rick Dreyfuss for assistance with IHC imaging; Udo Kontny and Joachim Baumgart for providing treosulfan; and Lee Helman and Su-Young Kim for critical review of the manuscript.

Received for publication June 19, 2009, and accepted in revised form June 2, 2010.

Address correspondence to: Chand Khanna, Head - Tumor and Metastasis Biology Section, Pediatric Oncology Branch, Center for Cancer Research, National Cancer Institute \& Comparative Oncology Program, Center for Cancer Research, National Cancer Institute, National Institutes of Health, 37 Convent Drive, Rm 2144, Bethesda, Maryland 20892, USA. Phone: 301.594.3406; Fax: 301.443.1838; E-mail: khannac@mail.nih.gov.
1. Eccles SA, Welch DR. Metastasis: recent discoveries and novel treatment strategies. Lancet. 2007; 369(9574):1742-1757.

2. Krishnan K, Khanna C, Helman LJ. The molecular biology of pulmonary metastasis. Thorac Surg Clin. 2006;16(2):115-124.

3. Welch DR. Technical considerations for study- ing cancer metastasis in vivo. Clin Exp Metastasis. 1997;15(3):272-306.

4. Henriquez NV, et al. Advances in optical imaging and novel model systems for cancer metastasis research. Clin Exp Metastasis. 2007;24(8):699-705.

5 . Khanna C. Novel targets with potential therapeutic applications in osteosarcoma. Curr Oncol Rep.
2008;10(4):350-358.

6. Rhim JS, Putman DL, Arnstein P, Huebner RJ, McAllister RM. Characterization of human cells transformed in vitro by $\mathrm{N}$-methyl-N'-nitro-Nnitrosoguanidine. Int J Cancer. 1977;19(4):505-510.

7. Khanna C, Prehn J, Yeung C, Caylor J, Tsokos M, Helman L. An orthotopic model of murine osteo- 
sarcoma with clonally related variants differing in pulmonary metastatic potential. Clin Exp Metastasis. 2000;18(3):261-271.

8. Khanna C, et al. Metastasis-associated differences in gene expression in a murine model of osteosarcoma. Cancer Res. 2001;61(9):3750-3759.

9. Barkan D, et al. Inhibition of metastatic outgrowth from single dormant tumor cells by targeting the cytoskeleton. Cancer Res. 2008;68(15):6241-6250.

10. Aslakson CJ, Miller FR. Selective events in the metastatic process defined by analysis of the sequential dissemination of subpopulations of a mouse mammary tumor. Cancer Res. 1992;52(6):1399-1405.

11. Borowsky AD, et al. Syngeneic mouse mammary carcinoma cell lines: two closely related cell lines with divergent metastatic behavior. Clin Exp Metastasis. 2005;22(1):47-59.

12. Ha L, et al. ARF functions as a melanoma tumor suppressor by inducing p53-independent senescence. Proc Natl Acad Sci U S A. 2007;104(26):10968-10973.

13. Lifsted $\mathrm{T}$, et al. Identification of inbred mouse strains harboring genetic modifiers of mammary tumor age of onset and metastatic progression.
Int J Cancer. 1998;77(4):640-644.

14. Wan X, Mendoza A, Khanna C, Helman LJ. Rapamycin inhibits ezrin-mediated metastatic behavior in a murine model of osteosarcoma. Cancer Res. 2005;65(6):2406-2411.

15. Werner S, et al. Preclinical studies of treosulfan demonstrate potent activity in Ewing's sarcoma. Cancer Chemother Pharmacol. 2008;62(1):19-31.

16. Cifone MA, Fidler IJ. Correlation of patterns of anchorage-independent growth with in vivo behavior of cells from a murine fibrosarcoma. Proc Natl Acad Sci U S A. 1980;77(2):1039-1043.

17. Chambers AF, et al. Steps in tumor metastasis: new concepts from intravital videomicroscopy. Cancer Metastasis Rev. 1995;14(4):279-301.

18. Khanna C, et al. The membrane-cytoskeleton linker ezrin is necessary for osteosarcoma metastasis. Nat Med. 2004;10(2):182-186.

19. Cameron MD, et al. Temporal progression of metastasis in lung: cell survival, dormancy, and location dependence of metastatic inefficiency. Cancer Res. 2000;60(9):2541-2546.

20. Al-Mehdi AB, Tozawa K, Fisher AB, Shientag L, Lee A,
Muschel RJ. Intravascular origin of metastasis from the proliferation of endothelium-attached tumor cells: a new model for metastasis. Nat Med. 2000;6(1):100-102.

21. MacDonald IC, Chambers AF. Breast cancer metastasis progression as revealed by intravital videomicroscopy. Expert Rev Anticancer Ther. 2006;6(9):1271-1279.

22. Themistocleous GS, et al. Three-dimensional type I collagen cell culture systems for the study of bone pathophysiology. In Vivo. 2004;18(6):687-696.

23. Siminski JT, Kavanagh TJ, Chi E, Raghu G. Longterm maintenance of mature pulmonary parenchyma cultured in serum-free conditions. Am J Physiol. 1992;262(1 pt 1):L105-L110.

24. Movat HZ. Demonstration of all connective tissue elements in a single section; pentachrome stains. AMA Arch Pathol. 1955;60(3):289-295.

25. Hsu SM, Raine L, Fanger H. Use of avidin-biotinperoxidase complex $(\mathrm{ABC})$ in immunoperoxidase techniques: a comparison between $\mathrm{ABC}$ and unlabeled antibody (PAP) procedures.J Histochem Cytochem. 1981;29(4):577-580. 